\title{
The Isolated and Cross-circulated AV Node Preparation of the Dog
}

\author{
Koroku Hashimoto, Toshiniko Iijima, Keitaro Hashimoto \\ and Norio TaIra \\ Department of Pharmacology and Experimental Therapeutics, \\ Tohoku University School of Medicine, Sendai
}

\begin{abstract}
Hashimoto, K., It.mma, T., Hashimoto, K. and Talra, N. The Isolated and Cross-circulated $A V$ Node Preparation of the Dog. Tohoku J. exp. Med., 1972, $107(3), 263-275$ - The isolated AV node preparation of the dog consists of the right atrium and ventricular septum, and thus includes the main conducting pathways from the sinoatrial node through peripheral Purkinje fibers. The preparation is cross-circulated with a donor dog through the cannulated right coronary, anterior septal (ASA), and posterior septal arteries (PSA). The preparation permits close-arterial injection of drugs into the AV nodal area selectively via either the PSA or the ASA. The preparation also permits direct visual placement of recording electrodes at desired sites along the main conducting system. The preparation runs for $12 \mathrm{hr}$ or more. Relation of $\mathrm{AV}$ conduction to rates of atrial pacing observed in this preparation is essentially the same as that observed in the isolated hearts. Occlusion of the PSA causes impairment of AV conduction, while that of the ASA causes only diminution of ventricular potentials. Acetylcholine causes a negative dromotropic effect and $l$-norepinephrine a positive one via both the PSA and the ASA. However, the two substances are more effective via the PSA than via the ASA.— AV conduction; acetylcholine; norepinephrine
\end{abstract}

Since atrioventricular (AV) conduction is one of common interests in the medical science, a considerable amount of information is available concerning effects of various drugs on AV conduction. However, AV conduction of the heart in situ is variously affected by many extracardiac factors, and results obtained from such preparation are not so simple to explain the mode of action of drug on AV conduction. Thus, elaborate pioneer works were performed by using the heart-lung preparation (Krayer et al. 1951) or the Langendorff preparation (Alanis et al. 1958). On the other hand, the recent development of the technique for the selective perfusion of the AV nodal area of the dog heart in situ through the posterior septal ("AV node") artery provided entirely new approach for the investigation of the mode of drug action of the AV nodal area (Nadeau and AmirJahed, 1965). This facilitated gain on physiological and pharmacological knowledges of the AV nodal area (Chiba et al. 1969a, b, Gauthier and Nadeau 1969, Chiba and Hashimoto 1970). However, for cannulation of the "AV node" artery, the posterior descending artery distal to the origin of the former must be ligated.

Received for publication, December 28, 1971. 
This results in ischemia in the posterior part of the ventricular myocardium, larger or smaller in its extent. Recently two of us (Hashimoto and Hashimoto $1971,1972)$ perfused in situ the anterior septal artery which supplies the ventricular septum without producing the myocardial ischemia.

We prepared the isolated $\mathrm{AV}$ node preparation of the dog heart. The preparation was essentially composed of the right atrium and the ventricular septum, and thus included the main conducting pathways from the sinoatrial node through peripheral Purkinje fibers. The preparation was cross-circulated with a donor dog through the cannulated right coronary, "AV node", and anterior septal arteries. In this paper the technique for making the preparation and the fundamental characteristics of its $\mathrm{AV}$ conduction are described.

\section{Methods}

\section{Atrioventricular (AV) node preparations}

Adult mongrel dogs weighing $6-8 \mathrm{~kg}$ were anesthetized with ether and exsanguinated from the cut right carotid artery after an i.v. injection of $200 \mathrm{U} / \mathrm{kg}$ of sodium heparin. After $300-400 \mathrm{ml}$ of blood were shed, the thorax was opened at the fifth right intercostal space and the heart was quickly removed. The excised heart was immediately immersed in Tyrode solution at about $4{ }^{\circ} \mathrm{C}$ and gassed with a mixture of $95 \% \mathrm{O}_{2}$ and $5 \% \mathrm{CO}_{2}$. In this circumstance the heart ceased the spontaneous beats. In most hearts the anterior septal artery (ASA) which supplies the ventricular septum submerged immediately into the myocardium after it branched off the anterior descending artery (ADA). Thus, cannulation of the ASA was performed as described previously by the two of us (Hashimoto and Hashimoto, 1971). The origin of the ASA was dissected free and a fine cotton ligature was placed at its origin. Then, the initial segment, $2-3 \mathrm{~mm}$, of the ADA was dissected free and a second ligature was tied. An incision of the artery was made proximally to the second ligature. A 13-guage polyethylene cannula tapered down to about less than $1 \mathrm{~mm}$ in diameter at the tip and filled with Tyrode solution was inserted into the ASA through the incision made on the ADA. The first and second ligatures were tied to fix the cannula. Since the posterior septal artery (PSA) which supplies the atrial margin of the AV node (James 1964, Nadeau and Amir-Jahed 1965) was usually too thin to permit direct cannulation, the PSA was cannulated indirectly through the left circumflex artery (LCA) of which branch the PSA is. The initial segment of the LCA, about $10 \mathrm{~mm}$, was dissected free and a polyethylene cannula similar to that to the ASA was inserted and fixed. The posterior descending artery, a main branch of the LCA, and other branches supplying the left atrium and the left ventricular free wall were ligated leaving the PSA alone intact. Since preliminary experiments showed that the right atrium and consequently the ventricular septum became unresponsive to electrical stimulation delivered at the crista terminal region unless the right coronary artery (RCA) was perfused with blood, the RCA was cannulated for perfusion. After the initial segment of the RCA, about $10 \mathrm{~mm}$, was dissected free and cannulated, its branches supplying the right ventricular free wall were all ligated. Then, the left atrium and the right and left ventricular free walls were removed leaving the ventricular septum. The right atrium was incised to expose the right aspect of the interatrial septum and the endocardial surface of the right atrium. Fig. 1 shows the accomplished AV node preparation with three cannulae in the RCA, ASA and PSA inserted.

The three polyethylene cannulae were connected with rubber tubing, each of which was joined together to a piece of polyethylene tubing. Arterial blood led from the cannulated carotid artery of a donor dog was made to flow into this tubing through an electromagnetic flow probe (Nihon Kohden, MF-2T) by means of a peristaltic pump (Harvard Apparatus, Model 600-1200). A pneumatic resistance was set in parallel with the perfusion circuit 


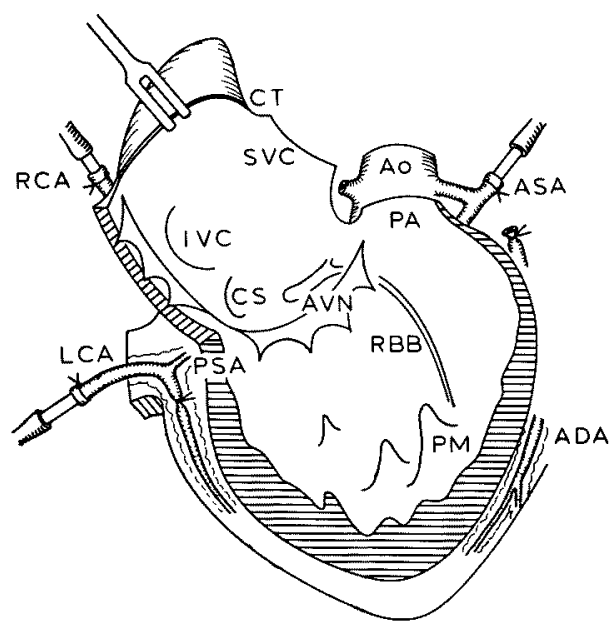

Fig. 1. The AV node preparation drawn schematically. The right coronary artery (RCA) and the anterior septal artery (ASA) are cannulated. The posterior septal artery (PSA) is cannulated via the left circumflex artery (LCA).

ADA: the anterior descending artery; Ao: the aorta; AVN: the atrioventricular node; CS: the coronary sinus; CT: the crista terminalis; IVC: the orifice of the inferior vena cava; RBB: the right bundle branch; PA: the orifice of the pumonary artery; PM: the anterior papillary muscle of the right ventricle; $\mathrm{SVC}$ : the orifice of the superior vena cava.

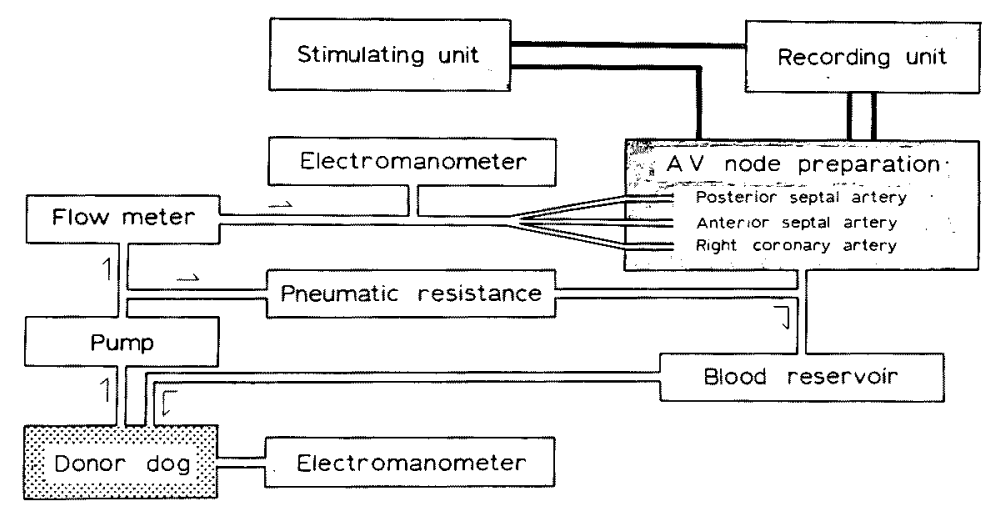

Fig. 2. Diagram of cross-circulation of the AV node preparation of the dog.

so as to maintain the blood flow at a constant pressure of $120 \mathrm{~mm} \mathrm{Hg}$. Total flow rate through the three cannulae was continuously monitored. Flow rate through each cannula was measured as reduction from the total flow rate when each rubber tubing was clamped for a brief period. Schematic diagram of cross-circulation of the AV node preparation is depicted in Fig. 2.

The preparation was placed in a funnel-shaped glass jacket through which water at about $40^{\circ} \mathrm{C}$ was circulated. The organ jacket was covered with a Lucite lid, and thus the surface of the preparation was kept moist and at $37-38^{\circ} \mathrm{C}$. Venous blood from the preparation was collected in the bottom of the jacket, led to a venous reservoir, and, in turn, returned to the donor dog via the cannulated jugular vein. 
As donors, mongrel dogs of both sexes, weighing $18-30 \mathrm{~kg}$, were anesthetized initially with an i.v. injection of $30 \mathrm{mg} / \mathrm{kg}$ of sodium pentobarbital. A constant level of anesthesia was maintained by supplementing $2-2.5 \mathrm{mg} / \mathrm{kg}$ of the same anesthetic every half an hour. The animals were heparinized with an initial dose of $250 \mathrm{U} / \mathrm{kg}$ of sodium heparin and supplemental ones of $100 \mathrm{U} / \mathrm{kg}$ every half an hour. Respiration of the animals was aided with a dog respirator (Harvard Apparatus, Model 607). The systemic blood pressure was monitored at the femoral artery by means of an electromanometer (Nihon Kohden, MP-2).

\section{Stimulating and recording device}

Stimulating electrodes consisted of two pieces of ball-tipped silver-silver chloride wire embedded in a small Lucite plaque keeping an interelectrode distance about $2 \mathrm{~mm}$. They were attached with fine silk sutures onto the endocardial surface of the crista terminal region. Rectangular pulses of 1-msec duration and voltage of two or three times the threshold voltage generated by an electronic stimulator assembly (Nihon Kohden, MSE40) were delivered through an isolation unit (Grass, SIU4A).

Recording electrodes were also an assembly similar to a stimulating one but consisted of three electrodes keeping interelectrode distances about $2 \mathrm{~mm}$ each other. The atrial recording electrodes were attached onto the endocardium of the free wall of the right atrium about $10 \mathrm{~mm}$ from the coronary sinus with fine silk sutures. The ventricular electrodes were located at the endocardium about $5 \mathrm{~mm}$ from the base of the anterior papillary muscle of the right ventricle toward the AV ring. This location usually permitted simultaneous recording of the electrical activity of the right bundle branch and that of the underlying myocardium. By monitoring visually action potentials on the screen of a monitor cathode-ray oscilloscope (Nihon Kohden, $\mathrm{VC}-7$ ), an appropriate pair of electrodes in each assembly were selected to pick up action potentials as simple and spike-like deflections.

The electrical activities thus picked up were displayed conventionally on the screen of a cathode-ray oscilloscope (Nihon Kohden, $\mathrm{VC}-6$ ) and finally recorded on X-ray film by means of a long-recording camera (Nihon Kohden, PC-1B). In most sorts of experiment, however, the shape of the action potentials of the right atrium, right bundle branch and ventricular septum remained practically unchanged. Therefore, we used an AV intervalgraph (Taira et al. 1971) for saving a laborious work to measure the conduction time. In addition, this device permitted us to observe continuously changes in the conduction time. Principle of the device is as follows: The action potential from the atrial electrodes and those of the right bundle branch and the underlying ventricular septum from the ventricular electrodes were amplified with two RC amplifiers with a time constant of 0.5 or 1 msec for a rising phase of each potential to be transformed to a pulse of short duration. After the outputs of the two RC amplifiers were mixed, each pulse was reshaped to a rectangular pulse of 0.5 - to 1-msec duration and of constant amplitude by a monostable circuit. The rectangular pulses were fed as brightening pulses to the Z-axis of one channel of a dualbeam cathode-ray oscilloscope (Nihon Kohden, VC-6). The beam of this channel was swept synchronously with each stimulus pulse and its intensity was adjusted so that bright spots emerged on the oscilloscope screen only when the brightening pulses were fed to the Z-axis. With this setup in each sweep, after the first spot corresponding to a stimulus artefact, spots corresponding to the rising phases of potentials of the right atrium, right bundle branch and ventricular septum emerged in succession on the oscilloscope screen with a suitable latency or conduction time. Fig. 3 shows photographic records obtained by using one beam of the oscilloscope to display either the atrial potential or the potentials of the right bundle branch and the ventricle in the conventional configuration, and the other beam as the $A V$ interval-graph.

Acetylcholine (ACh) chloride and $l$-norepinephrine $(l$-NE) base were used. ACh chloride was dissolved in $0.9 \%$ saline and $l$-NE base in $0.01 \mathrm{~N} \mathrm{HCl}$ to give a concentration of $1 \mu \mathrm{mol} / \mathrm{ml}$. ACh and $l \cdot \mathrm{NE}$ solutions thus made up were kept as stock solutions. Just prior to the experiment a series of concentrations were made by diluting the stock solutions 


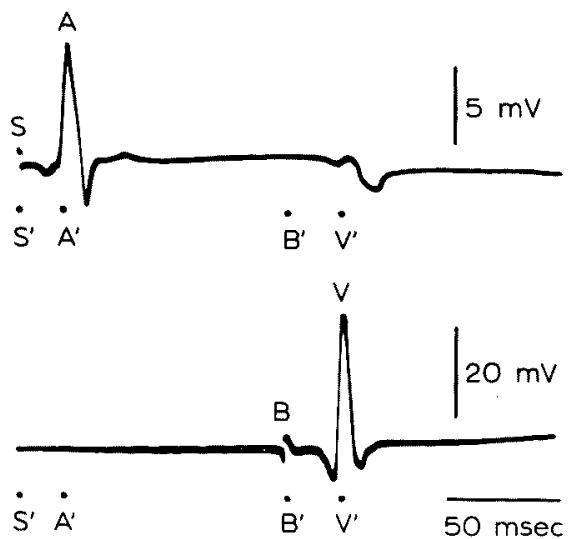

Fig. 3. Photographic records showing temporal relation of $A V$ interval-grams to original potentials picked up bipolarly from the right atrium, the right bundle branch, and the ventricular septum. Rising phases of stimulus artefacts (S), the potentials of the right atrium (A), of the right bundle branch (B), and of the ventricular septum (V) are converted by an $A V$ interval-graph to dots $S^{\prime}, A^{\prime}, B^{\prime}$, and $V^{\prime}$. Intervals $A^{\prime} B^{\prime}$, $\mathrm{B}^{\prime} \mathrm{V}^{\prime}$, and $\mathrm{A}^{\prime} \mathrm{V}^{\prime}$ are the time required by an impulse to travel from the right atrium to the right bundle branch, from the right bundle branch to the myocardium of the ventricular septum, and from the right atrium to the ventricular septum respectively.

with $0.9 \%$ saline. Injected volumes measured constantly $30 \mu \mathrm{l}$ and were injected at a constant rate for $4 \mathrm{sec}$ into the rubber tubing proximal to the artrial cannula with 50$\mu l$ microsyringes (Jintan Terumo)

\section{RESULTS}

Recovery process of rhythm and blood flow

Soon after cross-circulation started, the quiescent preparation regained its activity; the atrium began to beat spontaneously and the ventricular septum showed fibrillatory contractions. In 30 to $40 \mathrm{~min}$ most preparations resumed normal AV conduction. From this time on the preparations ran for 12 hours or more within which the scheduled experiment usually was finished.

Just after the commencement of cross-circulation total blood flow through the three cannulated arteries amounted up to 40 to $60 \mathrm{ml} / \mathrm{min}$ under a perfusion pressure clamped at $120 \mathrm{~mm} \mathrm{Hg}$. However, until normal AV conduction resumed, blood flow decreased gradually to a certain value and remained little changed for 12 hours or more. In this state the flow rate through the PSA was about $7 \mathrm{ml} /$ min, that through the ASA about $8 \mathrm{ml} / \mathrm{min}$ and that through the RCA about $5 \mathrm{ml} /$ min. When blood flow through the PSA decreased, AV conduction was readily impaired. Therefore, the effect of arterial occlusion on $\mathrm{AV}$ conduction was investigated. 


\section{Effect of occlusion of the PSA and of the ASA on $A V$ conduction}

In these experiments atrial and ventricular potentials alone were recorded. At a fixed stimulus interval of 400 msec the $\mathrm{AV}$ conduction time was in the range of 110 to $130 \mathrm{msec}$ and remained constant for a given preparation. Arterial occlusion was achieved simply by clamping with a pair of hemostats the rubber blood-conducting tubing just proximal to the arterial cannula. In all of five preparations occlusion of the PSA for 2.5 to 3 min caused impairment of $\mathrm{AV}$ conduction, although the severity of impairment depended on preparations. Upon occlusion the AV conduction time was little changed for the first one minute. However, in two of the five preparations for the following 1 to $1.5 \mathrm{~min}$ the $\mathrm{AV}$ conduction time was progressively prolonged by up to 50 to $60 \mathrm{msec}$ and finally AV block of Wenckebach type and 2:1 block took place. Even at this stage the configuration of ventricular potentials were not changed at all. In the remaining three preparations the AV conduction time was prolonged only by 10 to $30 \mathrm{msec}$. Upon release of occlusion AV conduction recovered within about $1.5 \mathrm{~min}$. An example of such five experiments is depicted in the left panel of Fig. 4 in which

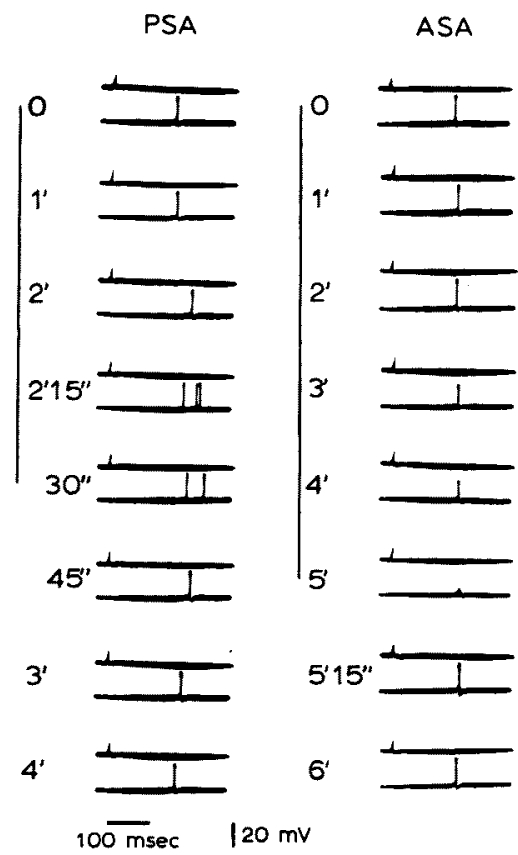

Fig. 4. Effects on AV conduction of occulsion of the posterior septal artery (PSA) and that of the anterior septal artery (ASA). The right atrium was driven at an interval of $400 \mathrm{msec}$. In each pair the upper records show the atrial and the lower ones the ventricular potentials. Each record is formed by superimposition of 12-13 traces. Periods of arterial occlusion are indicated by vertical bars. In this preparation occlusion of the PSA for $2.5 \mathrm{~min}$ caused prolongation of the AV conduction time, while that of the ASA for $5 \mathrm{~min}$ caused diminution of the ventricular potentials without significant prolongation of the $\mathrm{AV}$ conduction time. 
traces of atrial lead (upper traces of each pair) and those of ventricular lead (lower traces of each pair) were superimposed for 2 sec every 15 sec.

Occlusion of the ASA for 3 to 5 min, unlike that of the PSA, caused diminution of amplitude of the ventricular potentials for the late $2 \mathrm{~min}$ in two of the four preparations. Even in this stage the $\mathrm{AV}$ conduction time was not changed substantially. Upon release of occlusion the ventricular potentials regained their control amplitude within about $30 \mathrm{sec}$. The right panel of Fig. 4 shows such an example obtained in the same way and in the same preparation that is shown in the left panel. In the remaining two preparations neither amplitude of the ventricular potential nor the $A V$ conduction time was changed at all.

Relationship betueen stimulus intervals and $A V$ conduction

Relationship between stimulus intervals and the $\mathrm{AV}$ conduction time as measured in 10 preparations was presented in Table 1. Since the sinoatrial node was left intact, the sinus rhythm took over the driving rate when stimulus intervals

TABLE 1. Relation of $A V$ conduction time to stimulus intervals of atrial pacing

\begin{tabular}{c|c|c|c|c}
\hline \multirow{2}{*}{ Prep. No. } & \multicolumn{3}{|c}{ AV conduction time (msec) at stimulus interval (msec) of } \\
\cline { 2 - 4 } & 500 & 400 & 320 & 250 \\
\hline 1 & 106 & 108 & 110 & WB* \\
2 & $126(112)$ & $129(116)$ & $137(124)$ & WB \\
3 & $128(120)$ & $129(121)$ & $136(128)$ & WB \\
4 & 115 & 115 & 118 & WB \\
5 & $106(96)$ & $110(100)$ & $120(110)$ & WB \\
6 & $114(92)$ & $119(95)$ & $126(100)$ & $2: 1$ block \\
7 & $120(96)$ & $125(100)$ & $134(110)$ & WB \\
8 & 118 & 118 & 122 & $3: 1$ block \\
9 & $120(90)$ & $122(92)$ & $139(109)$ & WB \\
10 & $120(114)$ & $122(116)$ & $126(120)$ &
\end{tabular}

Time required by an impulse to travel from the right atrium to the right bundle branch is shown in parentheses.

* AV block of Wenckebach type.

were longer than 400 or $500 \mathrm{msec}$. As can be seen in this table, a marked increase in AV conduction time occurred at stimulus intervals between 400 and $320 \mathrm{msec}$. At a stimulus interval of $250 \mathrm{msec}$ various types of $\mathrm{AV}$ block took place.

\section{Effects on $A V$ conduction of $A C h$ injected into the PSA and the $A S A$}

In accord with results in the in situ AV node preparations (Nadeau and AmirJahed 1965, Hashimoto and Hashimoto 1971, 1972), when injected into the PSA, ACh caused a transient prolongation of the $\mathrm{AV}$ conduction time in doses of 0.01 to $1 \mathrm{nmol}$ and complete $\mathrm{AV}$ block in doses of 0.1 to $10 \mathrm{nmol}$. In these circumstances the configuration of the ventricular potential was not changed at all. In general, the shorter the stimulus interval, the more effective ACh in depressing $\mathrm{AV}$ conduction. Fig. 5 shows such an example in which intervals between trains 
of dots denoted as $\mathrm{A}$ and those of $\mathrm{V}$ present the $\mathrm{AV}$ conduction time.

Close-arterial injection of ACh into the ASA also caused a transient prolongation of the AV conduction time or AV block without change in the ventricular potential shape. When compared at the stimulus interval of $400 \mathrm{msec}$, however, doses of ACh required to match the negative dromotropic effect caused by $\mathrm{ACh}$ injected into the PSA were about 30 times as much as those injected into the PSA. Fig. 6 shows such an example obtained in the same preparation that was used in
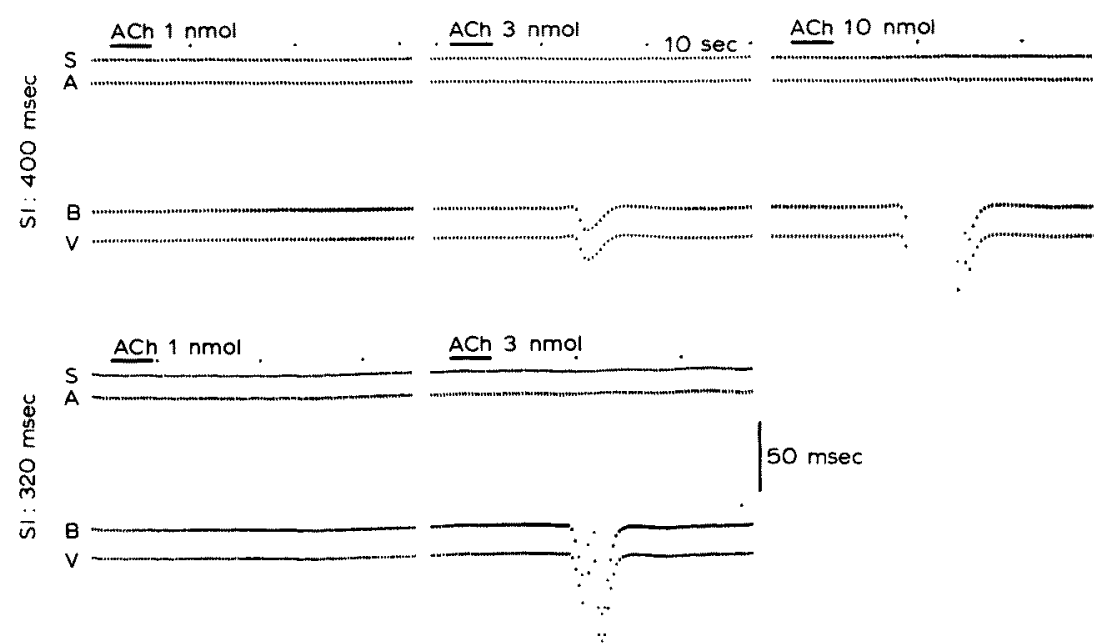

Fig. 5. AV interval-grams showing effect on $\mathrm{AV}$ conduction of acetylcholine (ACh) injected selectively into the PSA. Stimulus intervals (SI) of atrial pacing were 400 msec in upper panel and $320 \mathrm{msec}$ in lower one. S: stimulus artefacts of atrial pacing. Dots denoted as $A, B$, and $V$ present the time of arrival of impulses at recording sites of the right atrium, the right bundle branch, and the ventricular septum. Impairment of $\mathrm{AB}$ conduction by acetylcholine was pronounced at shorter stimulus intervals.

Fig. 5. It must be also noted that ACh injected into the ASA caused the positive dromotropic effect after AV block. When injected into the PSA, comparable doses of ACh scarcely caused a positive dromotropic effect after AV block.

\section{Effects on $A V$ conduction of $l-N E$ injected into the PSA and the ASA}

Close-arterial injection of $l$-NE in doses of 0.03 to $0.3 \mathrm{nmol}$ into the PSA caused a dose-dependent decrease in the AV conduction time when the atrium was paced at an interval of $400 \mathrm{msec}$. However, there occurred no change in the configuration of the ventricular potential. With doses larger than 0.1 to $1 \mathrm{nmol}$ the rhythm produced by the enhanced automaticity of the AV node took over the paced rhythm. In most preparations the $\mathrm{AV}$ nodal tachycardia took place at a time when the positive dromotropic effect reached its maximum. The left panel of Fig. 7 shows such an example. Despite uninterrupted atrial pacing disappearance of atrial potentials (dots denoted as A) indicates that the atrium was made 


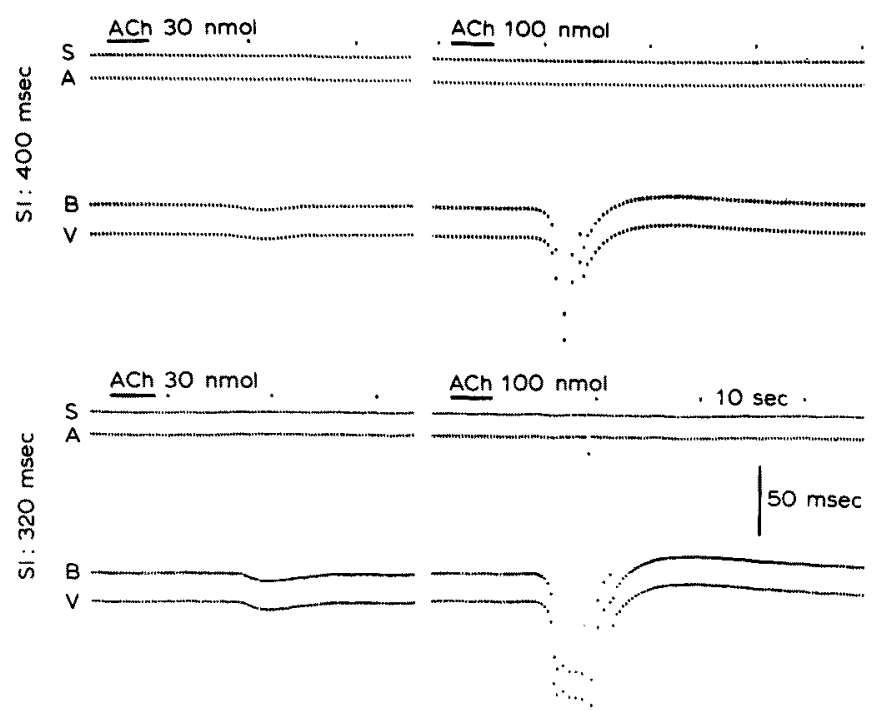

Fig. 6. $A V$ interval-grams showing effect on $A V$ conduction of acetylcholine (ACh) injected selectively into the ASA. Notations are the same as in Fig. 5. The negative dromotropic effect of acetylcholine was less dependent on stimulus intervals of atrial pacing.

refractory to atrial pacing by preceding impulses which invaded the atrium retrogradely from the $A V$ node. The automaticity of the $A V$ node enhanced by $l$-NE was readily suppressed by decreasing stimulus intervals (right panel of Fig. 7). The AV conduction time was longer at a stimulus interval of $320 \mathrm{msec}$ than at 400 msec. However, with each dose of $l$-NE the AV conduction time became shorter at a stimulus interval of $320 \mathrm{msec}$ than at $400 \mathrm{msec}$. In other words, the positive dromotropic effect of $l$-NE was more marked with pacing at 320 than at 400 msec.

Close-arterial injection of $l$-NE into the ASA also caused a positive dromotropic effect but no change in the ventricular potential shape. As with $\mathrm{ACh}$, doses of $l$-NE required to match the effect caused by $l$-NE injected into the PSA were about 30 times those administered into the PSA. Even with increasing doses up to $30 \mathrm{nmol}$ the $\mathrm{AV}$ nodal tachycardia was not produced but ventricular rhythm often took place.

Behavior of potentials of the right bundle branch and of the bundle-ventricular $(B V)$ conductim time

When the placement of the ventricular recording electrodes was appropriate, the spike-like deflection of 20 to $30 \mathrm{mV}$ signaling excitation of the ventricular septum at the recording site was preceded by a spiky deflection of a few millivolts at intervals of 8 to $14 \mathrm{msec}$ (Fig. 3). These intervals varied from preparation to preparation in that range. But for a given preparation the interval remained 


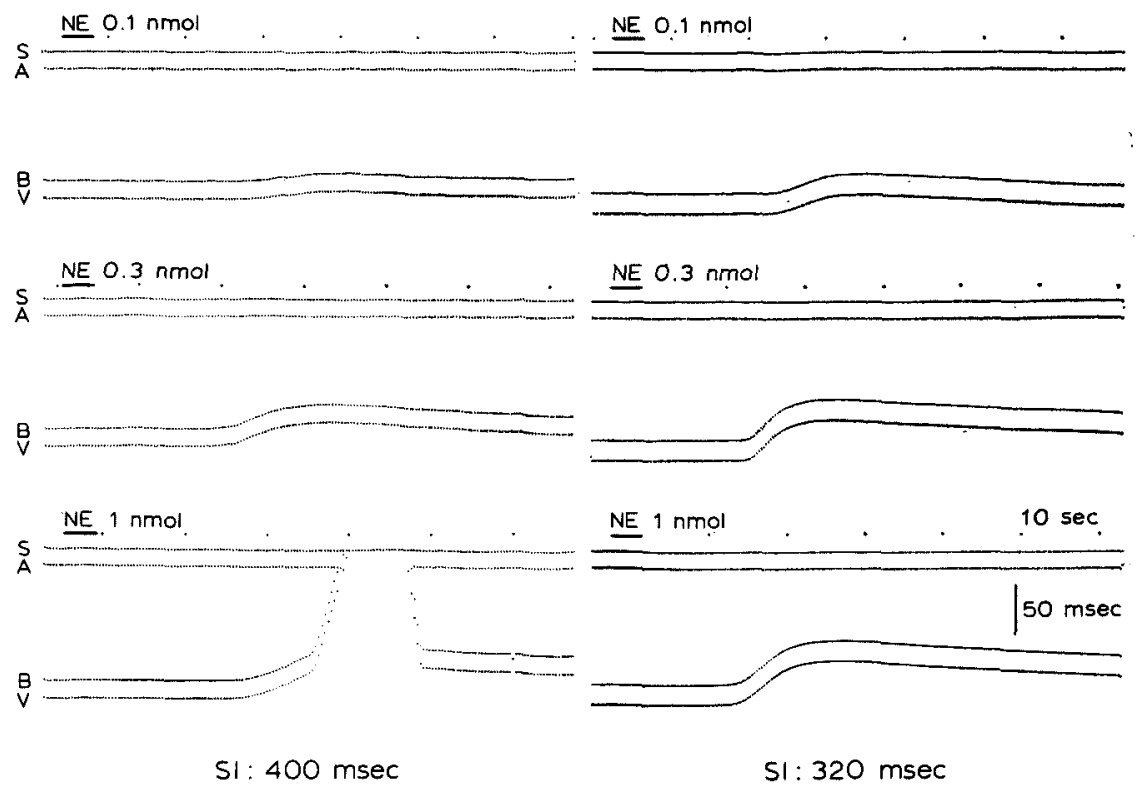

Fig. 7. AV interval-grams showing effect on $\mathrm{AV}$ conduction of $l$-norepinephrine (NE) injected selectively into the PSA. l-Norepinephrine caused a dose-dependent decrease in the $\mathrm{AB}$ conduction time, and consequently that of the AV conduction time. At a stimulus interval of $400 \mathrm{msec} 1 \mathrm{nmol}$ of $l$-norepinephrine caused so-called AV nodal tachycardia of which cycle length was shorter than 400 msec but longer than 320 msec.
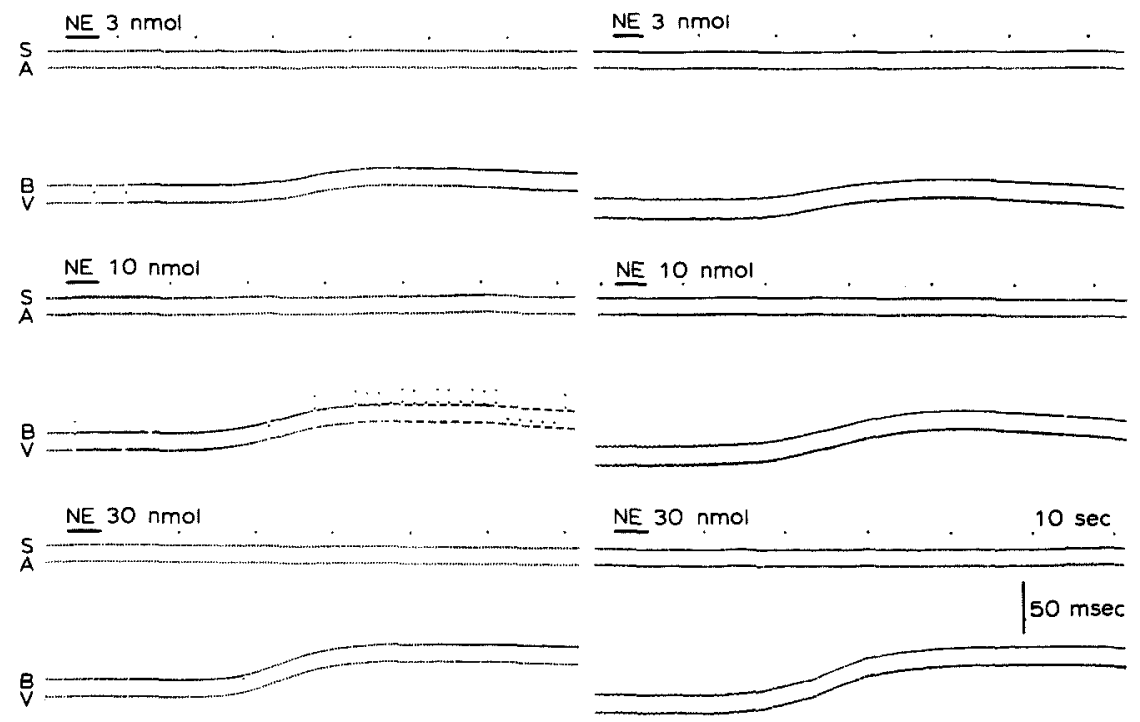

$\mathrm{SI}: 400 \mathrm{msec}$

$\mathrm{sl}: 320 \mathrm{msec}$

Fig. 8. AV interval-grams showing the positive dromotropic effect of $l$-norepinephrine injected into the ASA, 
constant. Judging from the location of the recording electrodes in reference to anatomical landmarks and comparing the configuration and temporal relation of the potential to the atrial and ventricular potentials with those obtained by previous workers (Burchell et al. 1953, Hoffman et al. 1959, 1960), the small spiky deflection is considered to be the electrical activity of the right bundle branch.

With alteration of stimulus intervals, the time required by an impulse to travel from the atrium to the right bundle branch ( $\mathrm{AB}$ interval) was changed in parallel with the AV interval. But, the time required by an impulse to travel from the right bundle branch to the ventricular septum (BV interval) was not changed significantly (Table 1).

Upon injection of $\mathrm{ACh}$ and $l$-NE into the PSA the bundle potential behaved exactly like the ventricular potential. As can be seen in Figs. 5 and 7, AB intervals changed in parallel with $A V$ intervals, while $B V$ intervals remained unchanged.

When $\mathrm{ACh}$ and $l$-NE were injected into the ASA, AB intervals changed in parallel with AV intervals as was the case of injection into the PSA (Figs. 6 and 8).

\section{Discussion}

In the present preparation values obtained as the AV conduction time with pacing the right atrium at stimulus intervals of 500 and $400 \mathrm{msec}$ approximated those obtained in the dog heart-lung preparation (Krayer et al. 1951) and in the dog Langendorff preparation (Alanis et al. 1958). Furthermore, the relation of the $\mathrm{AV}$ conduction time to stimulus intervals observed in the present preparation also resembled that observed in the dog Langendorff preparation (Alanis et al. 1958). Thus, it is not unreasonable to consider that AV conduction of the present preparation represents normal conduction in the state free from the tonic neural influence. The preparation ran for 12 hours or more with such normal AV conduction.

The present preparation permitted us selective administration of drugs into either the RCA, the ASA, or the PSA. Furthermore, the preparation permitted us the direct visual placement of recording electrodes at any position along the conducting pathways, as the Langendorff preparation (Alanis et al. 1958) or the dog on cardio-pulmonary bypass (Hoffman et al. 1959, 1960) did. The simultaneous recording of the electrical activities both from the right bundle branch and from the underlying myocardium, for example, revealed the differential effects on $\mathrm{AB}$ and $\mathrm{BV}$ conduction of $\mathrm{ACh}$ or $l$-NE given into the PSA and of $\mathrm{ACh}$ or $l-\mathrm{NE}$ given into the ASA. Thus, the systematic location of recording electrodes in combination with the selective administration of drugs will facilitate more detailed analysis of the AV node activity.

Occlusion of the PSA and the ASA exerted differential effects on AV conduction in the present preparation. Occlusion of the PSA caused impairment of AV conduction ranging from simple prolongation of the $\mathrm{AV}$ conduction time to Wenckebach block or $2: 1$ block. This indicates that the structures directly concern- 
ing major AV delay are nourished predominantly by the PSA, although the AV nodal area has been reported to receive dual supply from the PSA and ASA (Lumb et al. 1959, James 1964). In this respect the present results appear in disaccord with the results obtained by Nadeau and Amir-Jahed (1965) in the in situ AV node preparation that occlusion of the PSA caused no change in the PR interval. It is presumable that more anastomoses exist between the PSA and ASA in the in situ heart than in the excised AV node preparation. Lumb et al. (1959) reported that the ligation of the ASA caused severer impairment of cardiac function than that of the PSA. As a matter of fact, in the present experiments the amplitude of the potentials of the ventricular septum was greatly reduced upon occlusion of the ASA, although the AV conduction time was not prolonged substantially. If occurred in the in situ heart, cease of excitation of the upper septum might lead to the fatal cardiac dysfunction. In this regard the present results are not so different from those of Lumb et al. (1959).

In accord with the results obtained by arterial occlusion, both ACh and $l$ NE injected into the PSA was more effective in modifying AV conduction than those given into the ASA. This is the confirmation of the results obtained by the two of us (Hashimoto and Hashimoto 1971, 1972) with selective perfusion of the PSA and the ASA in situ. However, it must be noted that there are some qualitative differences in their effects depending on the route of administration. Even with increasing doses, $l$-NE given via the ASA failed to cause the AV nodal tachycardia, and ACh given via the PSA scarcely caused a positive dromotropic effect after AV block, as was observed on injection into the ASA. This reflects differences in structures which drugs reach via the two routes, as the anatomy of blood supply to the AV nodal area by the two arteries tells (Lumb et al. 1959, James 1964); the PSA supplies the AV junctional area and the upper part of the AV node, while the ASA the lower.

\section{Acknowledǵment}

The present study was aided by grants from the Pharmacological Research Foundation, Inc., Tokyo, and Tokyo Research Laboratory, Kowa Company.

\section{References}

1) Alanís, J.H., Gonzáles, H. \& López, E. (1958) The electrical activity of the bundle of His. J. Physiol., 142, 127-140.

2) Burchell, H.B., Essex, H.E. \& Lambert, E.H. (1953) Action potentials supporting the presence of specialized conduction pathways in the dog's ventricle. Circulat. Res., 1, 186-188.

3) Chiba, S. \& Hashimoto, K. (1970) Blocking of acetylcholine-induced fibrillation by use of norepinephrine into the AV node artery. Jap. J. Physiol., 20, 560-570.

4) Chiba, S., Hashimoto, K. \& Hashimoto, K. (1969) Ventricular fibrillation induced by saxitoxin into the $A V$ node artery and its prevention by phenoxybenzamine. Tohoku J. exp. Med., 99, 103-104.

5) Chiba, S., Ohkuda, K. \& Hashimoto, K. (1969) Oscillatory nature of AV node pacemaker activity. Tohoku J. exp. Med., 97, 187-189. 
6) Gauthier, P. \& Nadeau, R.A. (1969) Effects of nicotine injected into the atrioventricular node artery of the dog. J. Pharmacol. exp. Therap., 169, 298-307.

7) Hashimoto, K. \& Hashimoto, K. (1971) Direct perfusion of the canine ventricular septum via the anterior septal artery. Tohoku J. exp. Med., 105, 99-100.

8) Hashimoto, K. \& Hashimoto, K. (1972) Cardiac irregularities induced by intracoronary injection of epinephrine and acetylcholine into various portions of the canine ventricle. Amer. Heart. J., 83, 197-205.

9) Hoffman, B.F., Cranefield, P.F., Stuckey, J.H., Amer, N.S., Cappelletti, R. \& Domingo, R.T. (1959) Direct measurement of conduction velocity in in situ specialized conducting system of mammalian heart. Proc. Soc. exp. Biol. Med., 102, 5557.

10) Hoffman, B.F., Cranefield, P.F., Stuckey J.H. \& Bagdonas, A.A. (1960) Electrical activity during the P-R interval. Circulat. Res., 8, 1200-1211.

11) James, T.N. (1964) Anatomy of the AV node of the dog. Anat. Rec., 148, 15-27.

12) Krayer, O., Mandoki, J.J. \& Mendez, C. (1951) Studies on veratrum alkaloids. XVI. The action of epinephrine and of veratramine on the functional refractory period of the auriculo-ventricular tramsmission in the heart-lung preparation of the dog. J. Pharmacol. exp. Therap., 103, 412-419.

13) Lumb, G., Shacklett, R.S. \& Dawkins, W.A. (1959) The cardiac conduction tissue and its blood supply in the dog. Amer. J. Path., 35, 467-487.

14) Nadeau, R.A. \& Amir-Jahed, A.K. (1965) Selective perfusion of the AV node of the dog by cannulation of the posterior septal artery. Rev. Canad. Biol., 24, 291297.

15) Taira, N., Iijima, T., Hashimoto, K. \& Hashimoto, K. (1971) A device for automatic plotting of the atrioventricular conduction time. Tohoku J. exp. Med., 105, 391395. 